\title{
Automatic Object Painting with SCARA Robot Using Computer Vision
}

\author{
İbrahim Kesici ${ }^{1}$, Şeyma Bişkin² ${ }^{2}$ Alaa Eleyan ${ }^{3 *}$ \\ ${ }^{1}$ Department of Automatic Control and Robotics Engineering, Warsaw University of Technology, Warszawa, Poland \\ ibrahim.kesici@outlook.com \\ ${ }^{2}$ Department of Power Engineering, Warsaw University of Technology, Warszawa, Poland, \\ biskinseyma@gmail.com \\ ${ }^{3}$ Department of Electrical \& Electronics Engineering, Avrasya University, Trabzon, Turkey, \\ aeleyan@avrasya.edu.tr \\ *Corresponding author
}

Received: 19 April 2017

Accepted: 5 March 2018

DOI: $10.18466 /$ cbayarfbe. 306950

\begin{abstract}
Recognizing and accurately classifying colors in industrial applications is a critical challenge in developing robotics painting applications. To achieve this, many painting robots are attached with expensive color sensors. However, these sensors are coming with some drawbacks such as color ranges limitation and sensitivity to illumination in addition to their high costs. In the last decades, camera systems gained importance in robotics applications with the power presented by the computer vision techniques. The main objective of this paper is to design an automation line that includes a robot and camera system to perform painting in different colors, with various illumination conditions at cheaper costs. The proposed system can be used to paint multiple colors effectively and accurately. The power of the system comes from the color detection and classification algorithm that is designed using computer vision techniques. The algorithm is designed under $\mathrm{C}++$ environment using OpenCV library. The system will able to detect all colors that are adjusted/predefined offline by the user and to work in different illumination conditions. The end-effector of the robot consists of two main parts, a camera to detect the desired color and an automatic spray gun to perform the painting operation. The proposed color detection system will be based on a small sticker pasted on the object that will be painted. When the desired color is detected, the system starts the painting operation. Moreover, the system has the capability to automatically cleaning the spray gun and the connected tubes in the case that the successive object is to be painted with different color.
\end{abstract}

Keywords - Automatic Painting, Color Detection, Computer Vision, Painting Robot

\section{Introduction}

The painting process requires high accuracy, repeatability and precise control during the operation. It is difficult to obtain homogeneously painted objects even with welltrained workers that have limited production capacities for a day. As educated workers must be hired for this, this cost constitutes a significant part of the entire production cost. To reduce the labor cost and to degrade the failure rate its minimum rate, automated robotic systems for painting process were proposed which meet highly accurate and sustained necessities of manufacturing [1-4]. However, production lines that apply paintings with various colors need good quality color sensors. Due to both high costs and color detection limitations of these sensors, forming such an automated system scales up the expenses. Moreover, most of the color sensors available in today's market come with restricted color ranges and are very sensitive to illumination and reflection conditions. Therefore, the camera systems offer attractive solutions to the color detection problem. During the last decade, camera systems gained importance in many areas of robotics from humanoid robots to industrial manipulators [5-7]. Recorded or real-time videos and images are processed using computer vision algorithms. OpenCV library in $\mathrm{C}++$ environment is very helpful tool in accomplishing the required tasks. It has been used in many applications such as object tracking by color [8], road sign recognition [9], and industrial classification areas [10].

The overall objective of this study is to conceive an automated painting system that has the capability of painting objects using different colors by means of a standard low-cost camera, an advanced color detection and classification algorithm, and an industrial robot. The usage 
of the proposed algorithm is expected to provide fast and accurate color information even in different illumination circumstances and make a connection between camera, automation line, and the robot.

This paper is organized as follows: In Section 2 the working mechanism of the whole system, materials and methods are described briefly in terms of the automation system and detection algorithm. In Section 3, results and simulation of the running system are presented. Finally, in Section 4, the conclusion is drawn, and suggestions are made for further developments.

\section{Materials and Methods}

\subsection{Experimental Setup}

\subsubsection{4-DOF SCARA Robot Arm}

Four degrees of freedom (DoF) EPSON LS3-401S model SCARA robot is used in the system design. The first two degrees of freedom of the robot directly affects maneuver while the last two joints are responsible for the painting process. The end-effector is designed to involve both the camera and automatic spray gun with an angle of 90 degrees apart. This is to ensure that the painting will not cover the camera during the painting process. The endeffector is attached to the last revolute joint of the robot. Figure 1 illustrates the end-effector's 2D and 3D designs and its connection to the last joint of the robot.

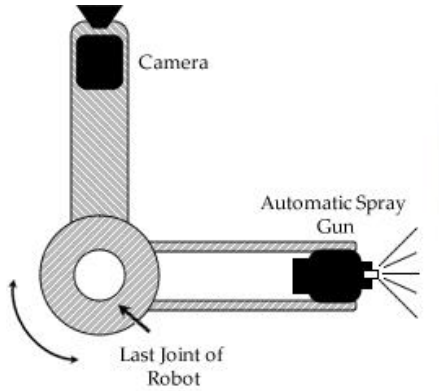

(a)

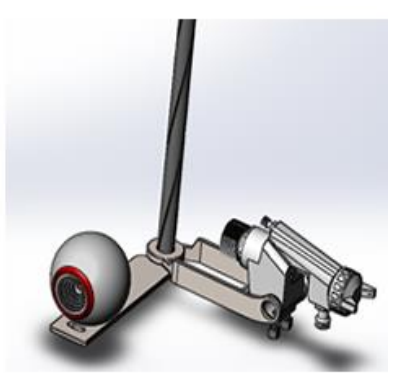

(b)

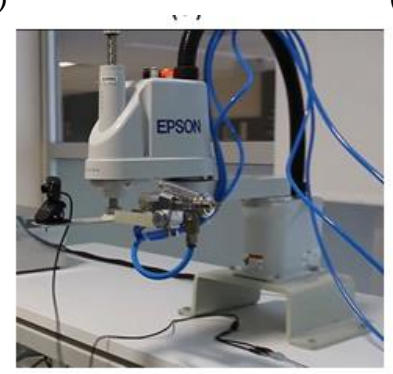

(c)

Figure 1. End-effector which holds the camera and automatic spray gun: (a) 2D Design, (b) 3D Design, (c) Real Design

The robot is programmed using Epson's SPEL+ official programming language and it has an I/O communication with Programming Logic Controller (PLC). The robot executes painting process based on the input signals that come from PLC.

\subsubsection{Pneumatic Painting System}

Five 2 liters - 6 bars pressurized paint tanks are used in the painting system. Four of the tanks contain recognized paint while one tank is filled with alcohol to clean the pipes after each color alterations and pressure of the tanks are arranged to 3 bars. On/Off solenoid valves are connected to the top of each paint tanks to electronically control which paint will be performed based on the coming signals from PLC. The painting system section designed based on two main considerations to obtain a clean painting. The first consideration, the connection pipes must be cleaned when successive painting colors are different. For this purpose, the tank which contains alcohol is situated at the first place. The compressed air in the compressor is cleaned and limited in FRL (Filter - Regulator - Lubricator) air regulator. This is to ensure that the pressure will not surpass the limits of the pressurized tanks at any time. The filtered air goes through the paint tanks from the alcohol tank to clean all pipes after each color alterations. The second consideration, automatic spray gun must homogenously repel the paint to the surface. An adjustable air regulator is used to regulate the spraying strength of spray gun. Moreover, a 3/2 directional solenoid valve with exhaust is connected to air input of spray gun to control it via PLC. Exhaust property is needed to release the air, inside the pipes, as soon as receiving a close signal from PLC.

\subsubsection{PLC and Arduino Controllers}

A PLC with an extension card is used both to control the painting process and communicate with the robot. PLC's five outputs are connected to the robot's controller and one output of the robot is connected to the input of the PLC. PLC undertakes three crucial operations in the setup. The first, it controls the conveyor belt's speed and informs the robot about the position of the object holder. Another operation of PLC is activation of both tanks' solenoid valves and spray gun's directional valve based on the signals coming from developed algorithm. The third, robot took actions depend on coming PLC signals. PLC notifies the robot about which actions must be taken and in which positions must be located at specific times.

The Arduino board is used to inform the PLC which paint must be performed based on the result of the computer vision algorithm. Arduino feeds PLC's four inputs based on the coming data from the proposed algorithm.

\subsubsection{Conveyor Belt}

Two meters long conveyor belt is used to move the pallet which carries the designed configurable object holder. Two proximity sensors are positioned to the conveyor belt. The first sensor is placed in front of the robot and a signal is sent to the PLC to stop the conveyor belt when the sensor detects 
the holder. After the completion of the painting operation, conveyor belt will continue to move till it reaches the second sensor. The second sensor is situated at the end of the conveyor belt to stop it as soon as it detects the holder.

\subsection{Color Detection Algorithm}

A custom image analysis algorithm is developed to process and interpret the color of a small sticker pasted on the object to send the related color character to the control system. The proposed algorithm is one of the major parts of the system since the accurate selection of the color for painting depends on its result. The implementation of the detection algorithm is based on the Opensource Computer Vision Library (OpenCV) and is written in $\mathrm{C}++$ language on Microsoft Visual Studio platform.

The detection process shown in Figure 2 represents the detailed procedure of the algorithm schematically. The detection algorithm consists of four phases. The first phase, calibration of desired color values, assigns desired colors to compare them with actual colors. This process should be done offline and can be adjusted at any time when the rearrangement or addition of new colors is needed. The second phase, thresholding operation using inRange OpenCV function, used to detect an object based on the range of pixel values it has. In the third phase, morphological operations, and histograms are both used to get rid of background noise and fill the small holes in the detected parts. The histogram is used to count white pixels on the image resulted from the morphological operations.

The last phase, sending detected color information, aims to send proper color characters to Arduino controller. Each captured image frames from the camera are converted from RBG color format to HSI (Hue - Saturation - Intensity) format. The goal of this conversion is separating the image intensity from the color information. Moreover, HSI color format provides convenience for both adjusting range of desired colors and overcoming the lighting variations due to external sources. In the following paragraphs, each phase of the image analysis algorithm is explained in more details.

\subsubsection{Phase I: Calibration of Desired Color Values}

The goal of the calibration phase is to arrange the lowest and the highest Hue, Saturation and Intensity components of each determined colors. The algorithm continues to detect colors based on these tuned HSI's components till the user changes them. Table 1 presents the predefined HSI color values that are used in the system design.

Table 1. The desired color ranges for different colors in HSI

\begin{tabular}{|c|c|c|c|c|}
\hline & Red & Green & Blue & Yellow \\
\hline H & $0-10$ & $44-98$ & $90-114$ & $15-179$ \\
\hline S & $66-255$ & $124-255$ & $100-231$ & $0-128$ \\
\hline I & $91-255$ & $0-255$ & $187-255$ & 255 \\
\hline
\end{tabular}

\subsubsection{Phase II: Thresholding Operation}

After the calibration phase, the threshold of the image in HSI format is performed by using OpenCV "inRange" function. Inrange thresholding differs from standard thresholding operation in two ways. Firstly, inrange performs threshold based on the range of pixel values while the standard thresholding implements fixed-level thresholding. Secondly, inrange can be applied to multichannels arrays in contrast to standard thresholding. Due to the use of the inrange function, the colored image's pixels which fail between the lowest and the highest ranges of HSI components are converted to white while the remaining pixels are transformed to black for each desired color and a binary image is obtained.

\subsubsection{Phase III: Morphological Operations \& Histogram}

Opening and closing morphological operations are essential to obtain an accurate and clean image frames. The thresholded binary image, can possess some undesirable noise because of illumination and/or other environmental factors. Opening operation is used to get rid of the background noise. Moreover, closing is used to fill the undesired small black holes appeared in the sticker itself. The number of white pixels in the final processed image is counted for each desired color by using histogram. Based on pixels count proper variables will be assigned. The color detection and classification process is illustrated in Figure 2.

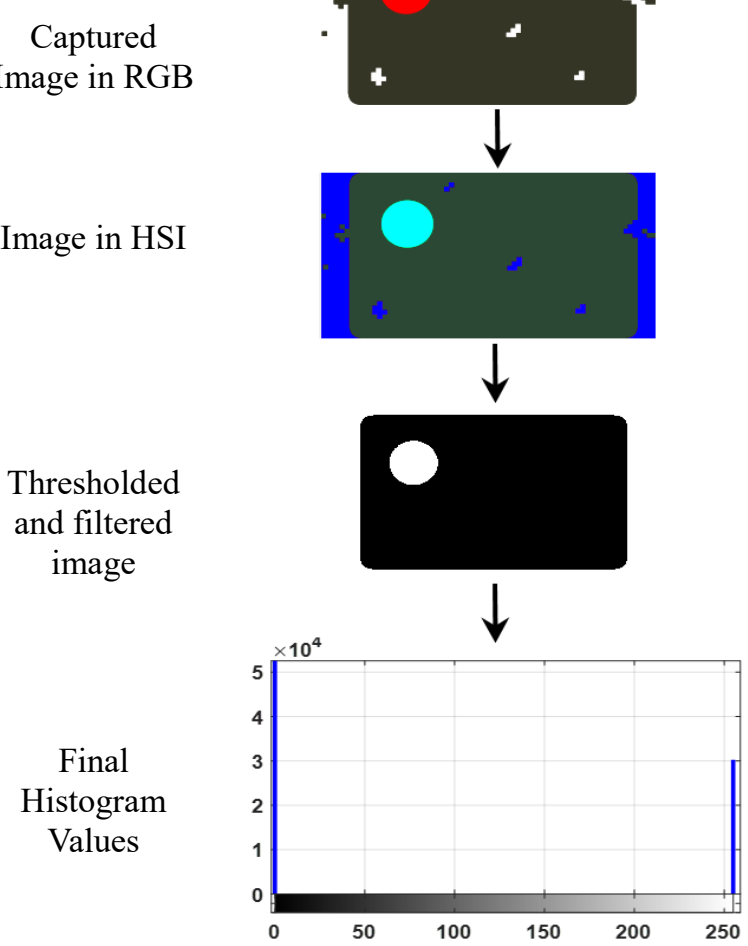

Figure 2. Phase III for color detection and classification algorithm. 


\subsubsection{Phase IV: Sending Detected Color Information}

The aim of this phase is to find the variable which contains the maximum white pixel values and sent this information to Arduino controller. The variable containing the maximum white pixels is declared by using a comparison algorithm.

As a result, we can detect the colors of the stickers by using designed algorithm. So, this obtained color information is sent to Arduino board as a character via the serial port.

\subsection{Summary of System Working Mechanism}

Figure 3 presents the schematic diagram of the whole system. Communication between SCARA Robot's RC90 controller, PLC, and Arduino controller is successfully established. As can be seen in the schematic, two proximity sensors are attached to the conveyor belt with the aim of detecting the position of the holder. Five solenoids valves are used to activate the pressurized color and cleaning tanks. Also, one 3/2 directional valve with exhaust is used to activate and deactivate the automatic spray gun. FRL is connected to the output of the $25 \mathrm{lt}$ compressor to supply the system a clean, filtered and lubricated air. The main aim of the PLC is to bridge between the robot and its environment. It controls the automation line and sends go home, paint, go paint position and activate camera signals to the robot controller. The system is driven in terms of four algorithms: First, the detection and classification algorithm which is written in $\mathrm{C}++$ language by using OpenCV library which detects and classifies the colors. Second, the algorithm in Arduino controller which links up $\mathrm{C}++$ code and PLC. Third, ladder diagram of PLC which controls the painting automation and send-receive signals from the robot. The last algorithm is designed in SPEL+ robot programming language to control the robot based on the signals that come from PLC.
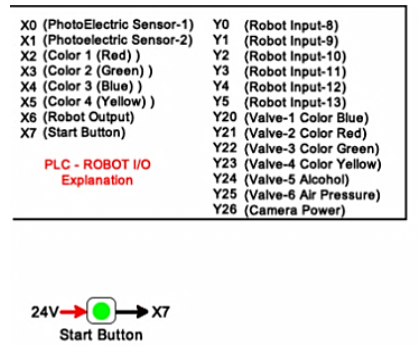

Start Button

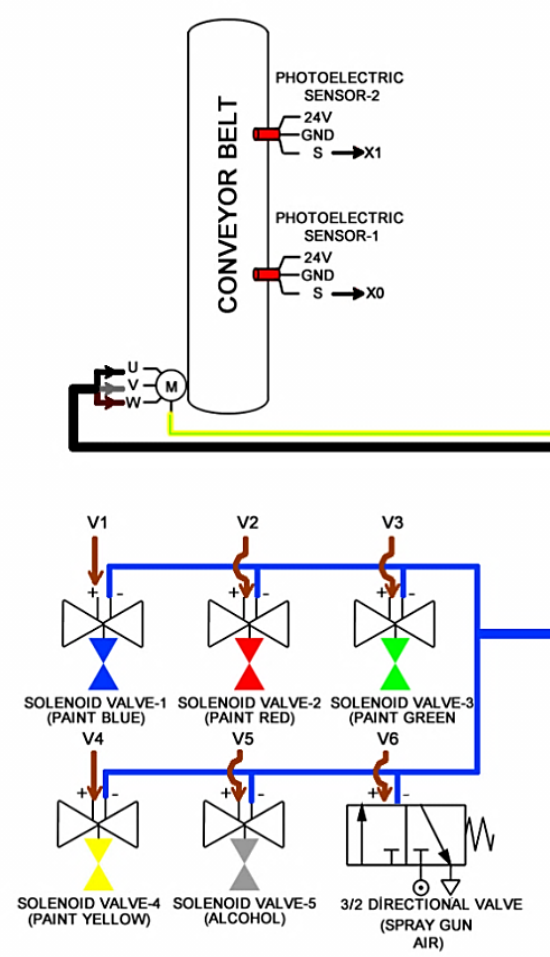

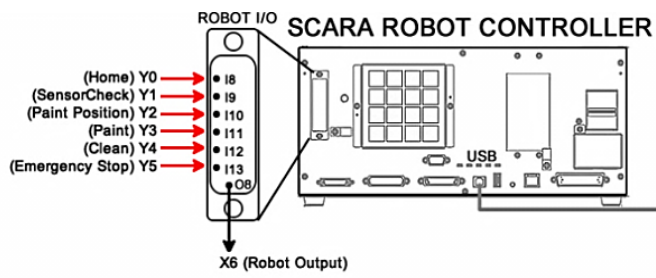
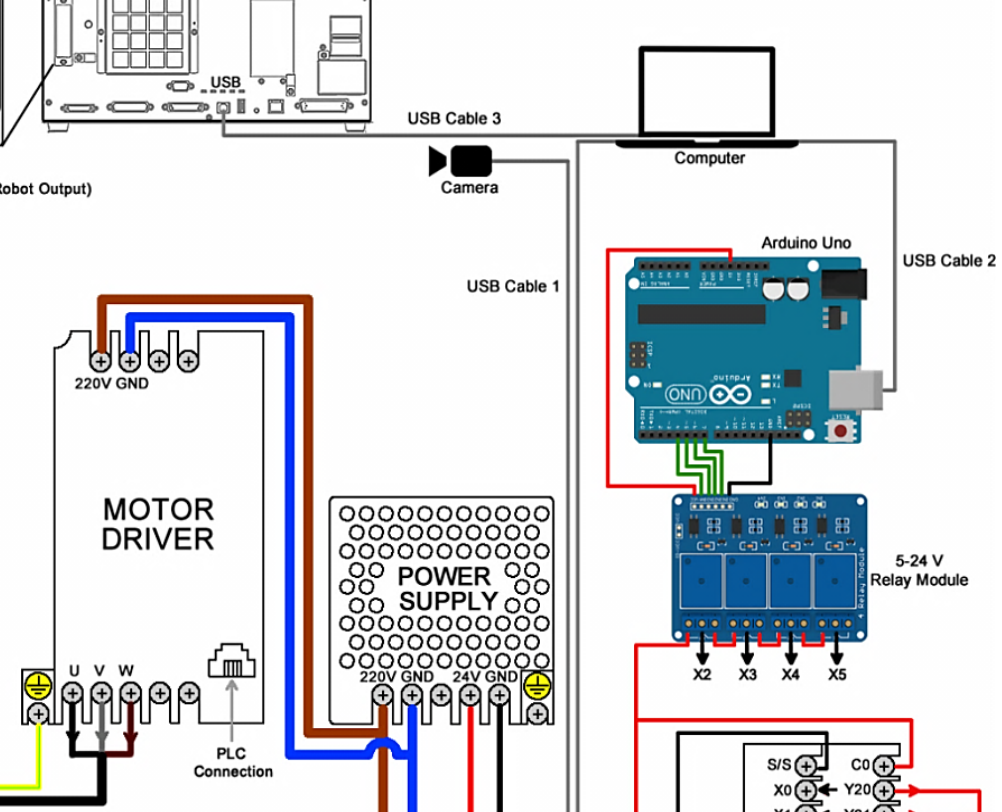

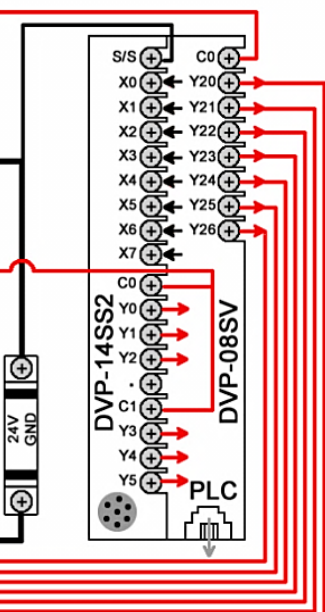

Figure 3 Schematic diagram for the whole system. 
The working strategy can be summarized as follows: The system is activated by pressing the start button and conveyor belt that carries the object starts to work. Conveyor belt continues to move till object reaches to predefined location in front of the robot. Proximity sensor will trigger the PLC to stop the conveyer belt. At this stage, the robot which is initially at the home position receives signal from PLC and takes a position that allows the camera to take a shot of the object. The camera is activated only when the robot takes such a position. This is to ensure that camera will only take the images of the relevant object. Advanced detection and classification algorithm assures that camera captures 20 images in a second. These images are processed, and algorithm decides the sticker's color based on the predefined HSI values (see Table 1). If there is no matching color with one of the predefined colors, the camera will take another 20 images for reprocessing. If the result is negative, the user will be notified, and automation will be paused. In the case of getting a match, this information is sent to Arduino controller. Arduino is then sends a signal, based on the recognized color, which is amplified on relays to the PLC. PLC uses this signal to activate related color solenoid valve and spray gun directional valve, and send it to the robot that will take the painting pose for operation. When the painting process is completed, the conveyor belt is activated, and it will run until the painted object comes to the end of the line. The algorithm will keep the painted color information to compare it with the next operation. If consecutive colors are different robot will take the cleaning position pose and spray gun will be cleaned. There will be no cleaning process if the successive objects with same colors.

\section{Results and Discussion}

Real-time simulation of the painting process is presented in Figure 4. The simulation is designed in Epson simulation platform by using the system's designed parts in SolidWorks and LS3 - 401S SCARA robot. The system is tested in several conditions before we begin the real design.

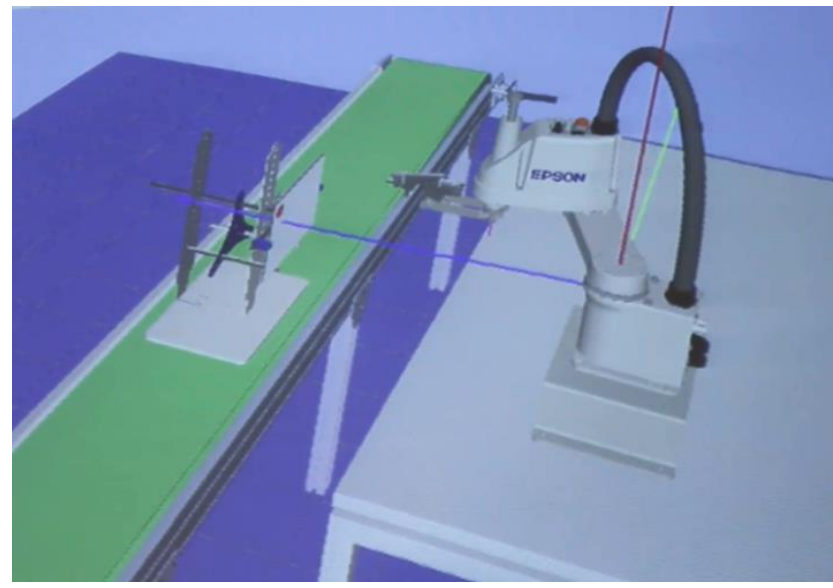

Figure 4. Real-time simulation of the painting process
The last joint of the robot is programmed to maintain perpendicular position to the object in the process and its trajectory is not showed because it makes minor movements during the painting process. Also, the first and seconds joints' trajectories are not included since they are only responsible for positioning. The only important joint for painting is the third joint. Figure 5 presents the trajectory of the robot's third joint. It illustrates one up-down cycle of the process. Robot repeats this up-down movement to obtain the painting. The distance that robot must travel, painting speed, spraying angle, and speed are calculated carefully.

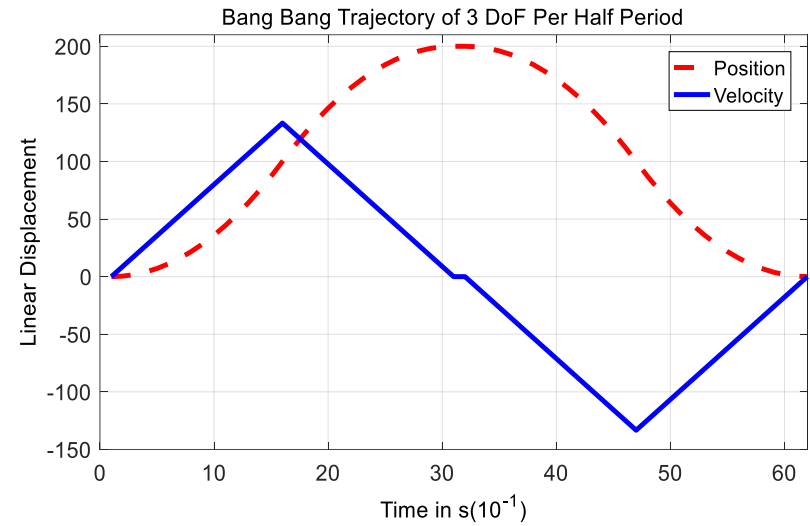

Figure 5. Trajectory planning of the robot's $3^{\text {rd }}$ joint

In the testing procedure, different scenarios are tested; stickers with the same colors are sent consecutively for detection. Stickers with different colors are sent consecutively and cleaning procedure is tested. Also, stickers in four different lighting conditions are tested. Even in dark environment, the LEDs mounted on the camera supplies enough illumination which helps running the system successfully. The system is also succeeded in reading the stickers with different angles. An advantage that color sensors lack it. As a result, we faced only one false detection (false negative) while the camera is reading the blue sticker. The algorithm achieved a total of $98.33 \%$ correct detection in different scenarios.

Some specific parts of the system such as end-effector and divider/object are manufactured based on drawings that are showed in Figure 6(a). The same drawings are also used in the simulation. Figure 6(b) shows the complete real-time system.

\section{Conclusions}

In this study, an advanced algorithm proposed and evaluated for detection and classification of small color stickers which are sticked on the objects that will be painted. The algorithm achieved $98.33 \%$ correct detection of all color stickers in different scenarios. Also, the algorithm showed that it can run successfully under different illumination conditions without losing its 
efficiency. This is due taking the average of the captured images and using the predefined ranges for detection. Furthermore, the proposed system can recognize the stickers in different angles while the available commercial color sensors such as ColorMAX 1000 are sensitive to angle variations. So, the errors that result of not placing the divider/object (color sticker) perpendicular to the sensor is eliminated without the need for any external devices.

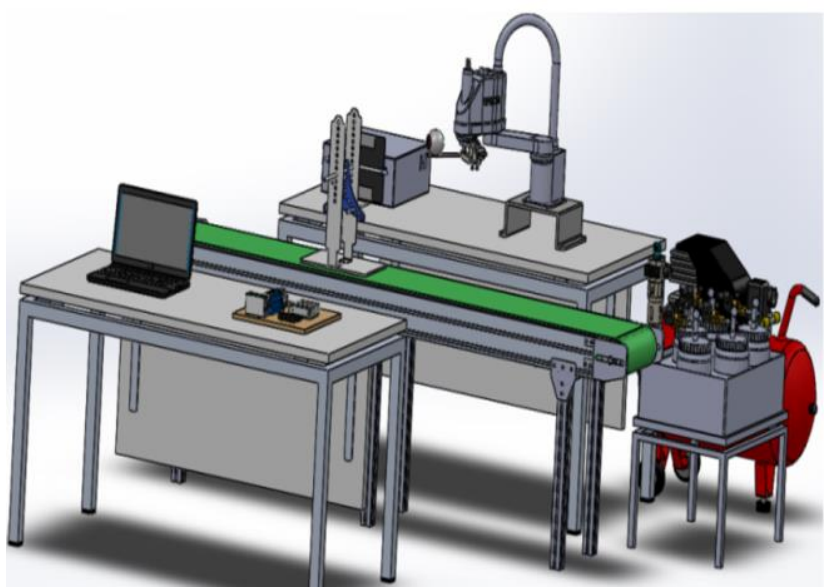

(a)

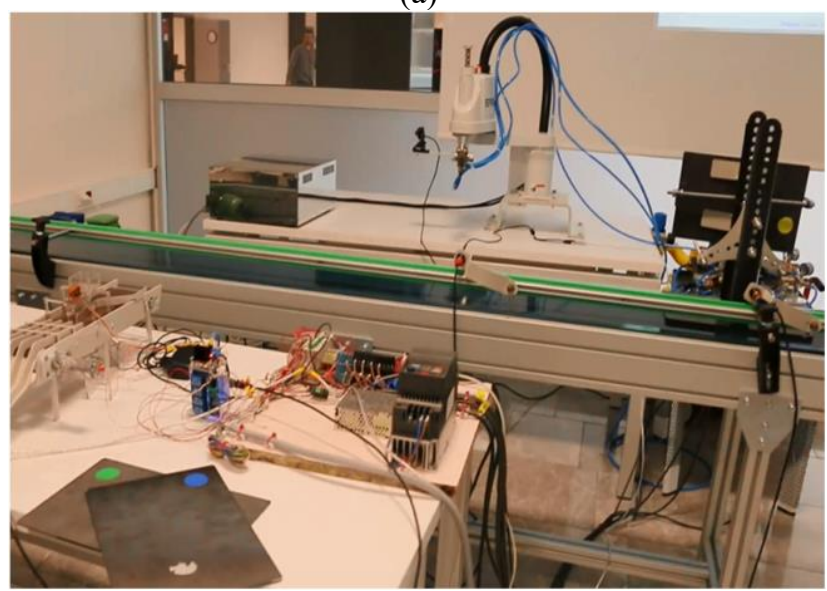

(b)

Figure 6. Automatic Painting System (a) SolidWorks system design, (b) complete real-time system

The main aim of the proposed design is to present more accurate and less expensive solution for detection of colors in painting process. A cheap web camera is used for detection operation instead of sophisticated and expensive color sensor. Moreover, the color limitations of the sensors are not an issue any more. The designed system can paint the objects quite easily in all colors which are predefined earlier by the user.

For future studies, we aim to enhance the system with shape detection and pose recognition capabilities and enable it to paint different shaped objects automatically.

\section{Acknowledgement}

Authors would like to thank Prof. Atef ATA for providing the resources of his previous TÜBITTAK project (Project No: 114E881) for conducting our experiments.

\section{References}

1. Ata, A, Eleyan A, Innovative Painting Robotic Cell for Industrial Applications, $3^{\text {rd }}$ International Symposium on Innovative Technologies in Engineering and Science (ISITES2015), Valencia, Spain, 2015, pp. $2462-2469$.

2. Ata, A, Eleyan A, An Autonomous Robotic Cell for Painting Applications, Proceedings of the IASTED International Conference Modelling, Identification and Control (MIC 2017), Innsbruck, Austria, 2017, pp. $200-207$.

3. Gasparetto, A, Vidoni, R, Pillan, D, Saccavini, E, Automatic Path and Trajectory Planning for Robotic Spray Painting, $7^{\text {th }}$ German Conference on Robotics, 2012, pp. 5925 - 5930.

4. Bo, Z, Fang, F, Zhenhua, S, Zhengda, M, Xianzhong, D, Fast and Template Path Planning of Spray Painting Robots for Regular Surfaces, $34^{\text {th }}$ Chinese Control Conference (CCC), 2015, pp. 211216.

5. $\mathrm{Mu}, \mathrm{J}$, and $\mathrm{Li}, \mathrm{Y}, \mathrm{A}$ New Efficient Real-Time Arbitrary Colored Ball Recognition Method for A Humanoid Soccer Robot, $12^{\text {th }}$ World Congress on Intelligent Control and Automation (WCICA), 2016, pp. $494-499$.

6. Shin, K, J, Detecting the Position of The Fish Robot Using the Color Segment Algorithm, Future Technologies Conference (FTC), 2016, pp. $896-900$.

7. Szabo, R, Gontean, A, Sfirat, A, Robotic Arm Control in Space with Color Recognition Using a Raspberry $\mathrm{Pi}, 39^{\text {th }}$ International Conference on Telecommunications and Signal Processing (TSP), pp. $689-692$, Dec. 2016

8. Silva, A,S, Marcolino, F, Severgnini, Q, Oliveira, M, L, Object tracking by color and active contour model s segmentation, IEEE Latin America Transactions, 2016, 1488 - 1493.

9. Russell, M, Fischaber, S, OpenCV Based Road Sign Recognition on Zynq, $11^{\text {th }}$ IEEE International Conference on Industrial Informatics (INDIN), 2013, pp. 596-601.

10. Constante, $\mathrm{P}$, Gordon, $\mathrm{A}$, Chang, O, Artificial vision techniques for strawberry's industrial classification, IEEE Latin America Transactions, 2016, 2576-2581. 